\title{
Lidil
}

Revue de linguistique et de didactique des langues

$51 \mid 2015$

Acquisition du langage et phonologie

\section{Acquisition du schwa en français (suisse) : pourquoi une combinaison de méthodes est-elle nécessaire pour assurer des données fiables?}

The Acquisition of Schwa Alternation in (Swiss) French: Why Is a Combination of Methods Required to Assure Reliable Data?

Helene N. Andreassen

\section{OpenEdition}

\section{Journals}

Édition électronique

URL : http://journals.openedition.org/lidil/3732

DOI : $10.4000 /$ lidil.3732

ISSN : $1960-6052$

Éditeur

UGA Éditions/Université Grenoble Alpes

Édition imprimée

Date de publication : 30 mai 2015

Pagination : 119-141

ISBN : 978-2-84310-297-4

ISSN : $1146-6480$

\section{Référence électronique}

Helene N. Andreassen, « Acquisition du schwa en français (suisse) : pourquoi une combinaison de méthodes est-elle nécessaire pour assurer des données fiables ? », Lidil [En ligne], 51 | 2015, mis en ligne le 30 novembre 2016, consulté le 01 mai 2019. URL : http://journals.openedition.org/lidil/3732 ; DOI : 10.4000/lidil.3732 


\title{
Acquisition du schwa en français (suisse) : pourquoi une combinaison de méthodes est-elle nécessaire pour assurer des données fiables?
}

\author{
Helene N. Andreassen*
}

\begin{abstract}
RÉSUMÉ
Malgré de très nombreux travaux sur le schwa, nous connaissons toujours peu son comportement lors de l'acquisition du langage. Cela pourrait être dû en partie à la manière de collecter des données fiables. Dans ce travail, nous poursuivons la discussion entamée dans Andreassen (2013), première étude détaillée sur le sujet. À la lumière de données de corpus suisses romandes, adultes et enfantines, nous concluons sur le fait qu'une combinaison de méthodes semble nécessaire pour révéler la richesse des stratégies adoptées par les enfants dans le processus d'acquisition de l'alternance schwa-zéro.
\end{abstract}

\begin{abstract}
Although schwa is a heavily studied phenomenon, we still know little about its behaviour in the course of language acquisition. One reason behind this lacuna could be the challenge of how to obtain reliable data. In this paper, we continue the discussion initiated in Andreassen (2013), the first detailed study on the subject. In light of Swiss French corpus data, from adult as well as child language, we conclude to the fact that a combination of methods in data collection seems required to reveal the rich selection of strategies used by the children before mastering target schwa alternation.
\end{abstract}

\section{Introduction}

En français, soit le schwa est réalisé avec une articulation proche de [œ] (ou [ø]) (voir, entre autres, Andreassen, 2013; Bürki, Racine, Andreassen,

* UiT Université arctique de Norvège. 
Fougeron \& Frauenfelder, 2008), soit il est absent de la forme phonétique, par exemple seconde [sœgõd]/[sgõd]. L'alternance du schwa a déjà été examinée sous une multitude d'angles, et ce par des chercheurs qui souscrivent à différents cadres théoriques et disciplinaires : phonologie générative ou modèles basés sur l'usage, phonétique acoustique, sociolinguistique, et, plus récemment, acquisition du français langue seconde (pour un tour d'horizon, voir Andreassen, 2013).

Jusqu'à présent, peu de travaux se sont concentrés sur le comportement du schwa dans l'acquisition du français langue première. $\mathrm{Si}$ Liégeois, Saddour et Chabanal (2012) et Liégeois (2014) examinent l'interaction parents-enfant et le taux d'alternance du schwa dans les monosyllabes, tels que je et que, Andreassen (2013) met davantage l'accent sur la structure segmentale dans l'output de l'enfant pour des items schwa polysyllabiques, tels que cerise et regarder. Dans ce travail, nous reprendrons la discussion entamée dans Andreassen (2013) sur l'enjeu méthodologique auquel tout travail sur l'acquisition de variables phonologiques doit faire face : quelle(s) méthode(s) sélectionner afin d'obtenir de données fiables, à savoir des données qui indiquent la compétence linguistique réelle de l'enfant?

Ce travail est organisé comme suit : la section 2 présentera les aspects du comportement du schwa dans le langage adulte pertinents pour cette discussion, alors que la section 3 sera consacrée à la méthodologie choisie. La section 4 présentera les résultats sur le comportement du schwa dans le langage enfantin, et finalement, avant de conclure, la section 5 contiendra une discussion des résultats à la lumière de la méthodologie adoptée.

Avant de présenter le schwa dans le langage adulte, nous mentionnons que nous utiliserons dans ce travail le terme «item schwa», qui est défini comme un mot polysyllabique dont la première syllabe est ouverte et contient un <e $>$ graphique, et dont la réalisation articulatoire est proche de [œ] (ou [ø]). Cette définition couvre alors des mots comme semaine, dont la voyelle $<\mathrm{e}>$ alterne, aussi bien que des mots comme semonce, dont la voyelle <e > n'alterne pas. Nous utiliserons également le terme «suite consonantique secondaire» (Bazylko, 1976) pour désigner les suites consonantiques qui sont créées lors de l'absence du schwa, par exemple [вn] dans renard [впаг].

Nous utiliserons également les termes «output»et «input». Si le premier réfère à la forme qui résulte du traitement phonologique et phonétique du mot, le deuxième réfère dans ce travail à la ou les variantes des items schwa auxquelles l'enfant est exposé. 


\section{Le schwa dans le langage adulte}

\subsection{Le langage inter-adulte}

Comme déjà indiqué dans les travaux de Martinet (1971) et Walter (1982), Racine (2008) confirme que l'alternance du schwa est sujette à la variation diatopique. En s'appuyant sur des données de jugement collectées auprès de deux groupes de locuteurs, provenant de Neuchâtel, en Suisse romande, et de Nantes, en France, Racine montre que la variante sans schwa est légèrement mieux acceptée dans la variété neuchâteloise que dans la variété nantaise. Si, pour certains items schwa, la différence inter-régionale n'indique qu'une gradation dans l'acceptabilité d'effacement du schwa (par exemple fenêtre reçoit des indices d'effacement indiquant une absence de schwa plus fréquente à Neuchâtel qu'à Nantes), pour d'autres items schwa, la différence inter-régionale indique un traitement phonologique différent du schwa : femelle reçoit par exemple des indices d'effacement indiquant une voyelle qui alterne à Neuchâtel, avec cependant une absence moins fréquente que dans fenêtre, mais une voyelle stable à Nantes, sans possibilité d'effacement.

La variation diatopique étant un fait, il s'ensuit que la compréhension de l'acquisition du schwa nécessite l'identification des taux d'acceptabilité des variantes avec et sans schwa dans la langue ambiante. Dans un premier temps, cette identification permet de préciser ce qu'est l'input de l'enfant, plus précisément ce que sont les taux d'alternance dans le lexique de son système cible. Dans un second temps, l'identification de ces taux permet d'identifier plus facilement le facteur dirigeant le choix de variante(s) dans l'output de l'enfant; ce choix reflète-t-il le comportement du schwa dans la communauté linguistique ou reflète-t-il plutôt des contraintes intra-grammaticales qui s'imposent à différents stades de l'acquisition phonologique?

Afin de déterminer la distribution du schwa dans le français suisse inter-adulte, l'auteure a examiné des données de jugement (Racine, 2008 ) et de production (tirées de la base du programme de recherche PFC : Phonologie du français contemporain, voir Durand, Laks \& Lyche, 2002, 2009a, <www.projet-pfc.net>). Cet examen, dont les détails peuvent être consultés dans Andreassen (2013), nous a entre autres permis d'identifier un groupe d'items schwa dont la voyelle n'alterne pas (ou peu), par exemple semonce (vs semaine) et pesant (vs pesé). Ce groupe est difficilement analysé comme le résultat de contraintes phonotactiques - par exemple, pourquoi y a-t-il alternance dans semaine mais pas dans semonce, alors que l'environnement consonantique est 
identique? Le groupe constitue donc, à priori, un défi pour l'enfant qui cherche à identifier le facteur linguistique qui gouverne l'alternance du schwa. Cependant, du moins dans nos corpus, nous observons qu'une grande partie de ce groupe d'items schwa est absente aussi bien du langage spontané de l'enfant que du langage adressé aux enfants (désormais LAE).

À cet égard, mentionnons Fikkert, Levelt et van de Weijer (2005), qui se positionnent dans le cadre de la théorie d'optimalité, et qui proposent l'émergence de contraintes de marque au cours de l'acquisition. Selon eux, l'enfant, arrivant au stade de segmentation de mot, généralise sur la base de son propre lexique. Étant donné que la nature de cette généralisation serait, du moins en partie, un reflet des patrons de fréquence dans la langue ambiante (Fikkert, Levelt \& van de Weijer, 2005), dans notre cas la fréquence des différents items schwa et celle de leurs variantes (avec et sans voyelle), nous estimons prudent, voire primordial, dans la formalisation grammaticale du phénomène, de ne prendre en compte que les items schwa réellement présents dans le langage enfantin.

\subsection{Le langage adressé aux enfants (LAE)}

Consciemment ou non, le LAE - le système adulte adapté à la conversation adulte-enfant - est souvent standardisé. Foulkes, Docherty et Watt (2005), Smith, Durham et Fortune $(2007,2009)$ et Smith, Durham et Richards (2013), parmi d'autres, montrent que, pour certaines variables linguistiques, les parents emploient davantage de formes «standards» lorsqu'ils s'adressent aux enfants. Les raisons sont multiples : désir d'assurer une plus forte clarté phonologique, de se rapprocher de la forme écrite ou d'utiliser la variante considérée comme socialement la moins stigmatisée. Une fois que l'enfant a acquis les deux variantes, les parents retourneraient aux normes inter-adultes (Smith, Durham \& Fortune, 2013; voir aussi Smith, Durham \& Fortune, 2007; Vosoughi, 2010); cependant, cet usage accru initial peut avoir des effets de longue durée sur la production linguistique de l'enfant : «[...] the standard [variant] may have more prominence in the speech of the caregiver and this is the form that the child will hear, and use, first.» (Smith, Durham \& Fortune, 2013, p. 317)

Quant au schwa, les travaux mentionnés ci-dessus indiquent que le LAE français contiendrait un taux plus élevé de la variante avec schwa comparé au langage inter-adulte, mais que l'usage accru de cette variante diminuerait de manière inversement proportionnelle au degré 
de maitrise du schwa de l'enfant. Ce scénario fournirait à l'enfant, du moins dans les premiers stades, une gamme d'exemplaires de la variante proche de la représentation phonologique supposée du mot, mais en même temps des taux d'alternance non représentatifs du système cible. De plus, l'absence du schwa étant le facteur principal permettant de séparer ce qui est un schwa de ce qui est un [œ] (ou [ø]) stable, que l'on trouve dans pleurer, par exemple, le LAE risquerait de ralentir la catégorisation de schwa $v s$ /oe/ dans la grammaire de l'enfant (pour une discussion plus détaillée, voir Andreassen, 2013).

Bien qu'il reste à confirmer que les enfants intègrent les patrons d'alternance des adultes avant de maitriser la complexité phonologique de l'alternance elle-même, nous estimons tout de même judicieux de prendre en compte le taux d'alternance dans le LAE lors de l'analyse globale de l'acquisition du schwa ${ }^{1}$.

Se focalisant sur le LAE, Liégeois, Saddour et Chabanal (2012) et Liégeois (2014) examinent le comportement du schwa des monosyllabes dans l'interaction parents-enfant. Sur la base de données spontanées enregistrées dans deux familles (les deux enfants âgés de 3;003;06 et 2;04-3;00 aux deux temps d'enregistrement) ${ }^{2}$, ils observent tout d'abord une plus forte présence du schwa dans le LAE comparé au langage inter-adulte. De plus, leurs travaux montrent que cette présence élevée du schwa est inversement proportionnelle au développement langagier de l'enfant. Ces résultats renforcent désormais la validité des travaux antérieurs, ainsi que l'hypothèse d'un comportement du schwa spécifique au registre que représente le LAE. Afin de tester cette même hypothèse sur des items schwa lexicaux - appartenant aux premières

1. Les travaux de Smith, Durham et Fortune $(2007,2009)$ et Smith, Durham et Richards (2013) montrent qu'une fois que les enfants savent produire les deux variantes, ils acquièrent rapidement les contraintes intra-grammaticales concernant leur usage - bien que le taux d'alternance puisse varier. Si cette observation nous est utile pour l'analyse du stade où l'enfant produit deux variantes, elle n'est pas indicative pour l'impact du LAE avant ce stade. Une observation dans nos propres données semble cependant indiquer une sorte de sensibilité au LAE : en se différenciant de nos autres locuteurs, et de manière quasi-obligatoire, Armand (cf. section 4) réalise cheval sans schwa dans son langage spontané. Le fait que sa mère est propriétaire d'une écurie fait penser que cet item schwa s'utilise souvent dans leur vie quotidienne, et ce sous sa forme vernaculaire - sans schwa.

2. Mentionnons que Liégeois (2014) inclut les données d'une troisième famille, dont l'enfant est âgé de 3;04-4;00 aux deux temps d'enregistrement. 
classes de mots abordées par l'enfant -, nous avons examiné le taux d'alternance du schwa dans le LAE de 6 femmes francophones, résidant sur la côte lémanique en Suisse. Bien que le corpus soit non équilibré et d'une taille réduite (1189 occurrences d'items schwa au total), et tout en admettant que nous n'avons pas pu entreprendre d'analyse statistique, l'examen quantitatif et qualitatif de nos données confirme la plus forte présence de schwa dans le LAE. Pour donner un exemple, la figure 1 compare le taux de présence de schwa dans le corpus LAE et le corpus PFC suisse (pour de l'information sur le corpus PFC, cf. ci-dessus), en contexte post-vocalique et en fonction de type de suite secondaire (pour des chiffres et d'autres détails, cf. Andreassen, 2013, p. 198-199).

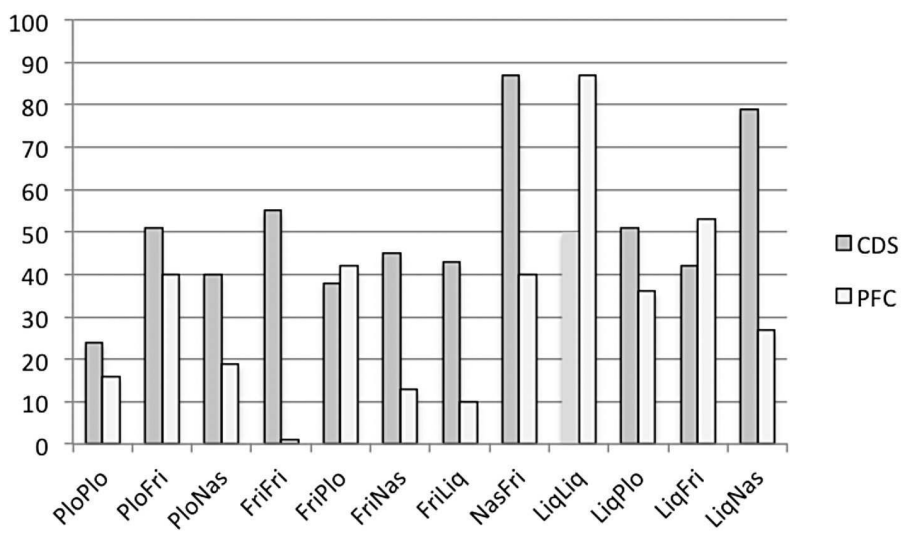

Figure 1. - Taux de présence de schwa en contexte post-vocalique, en fonction de type de suite secondaire, données conversationnelles des corpus LAE ( $=$ CDS, Child Directed Speech) et PFC.

Graphe tiré de Andreassen (2013, p. 198). Notons que la colonne LiqLiq de la catégorie LAE ne représente qu'une occurrence sur deux au total, et ne peut désormais pas être considérée comme représentative pour ce type de suite secondaire.

De plus, et conformément aux résultats de Roberts (1997) et Smith, Durham et Fortune (2007), qui examinent des variables linguistiques en fonction du niveau de formalité de la situation de communication, l'examen détaillé de deux de nos locutrices indique que la présence du schwa est d'autant plus importante dans des contextes communicatifs dits éducatifs (contexte Teaching) ou disciplinaires (contexte Discipline), 
comparés aux deux contextes communicatifs identifiés, Routine et Play (Andreassen, 2013; voir également Roberts, 1997; Smith, Durham \& Fortune, 2007, pour une introduction au système de codage contextuel). Les exemples présentés ci-dessous dans (1) illustrent l'item schwa sera produit par une de nos locutrices. Si elle produit le mot sans voyelle dans une situation jugée informelle (classifiée dans Routine), elle semble souligner l'importance du message transmis par la présence du schwa dans une situation jugée formelle (classifiée dans Discipline).

(1) Variation comportementale du schwa sur une échelle de formalité :

Mais nous quand Hélène $[$ sь] a partie, on ira où avec ton bateau?

Alice; destinataire Guy (3;06.13)

Contexte Routine

Absence de schwa

[La lolette], tu l'auras quand Hélène [sœs]a partie!

Alice; destinataire Guy (3;05.23)

Contexte Discipline

Présence de schwa

Si le LAE dévoile une plus forte contrainte sur la création de suites secondaires comparé au langage inter-adulte, cette différence interregistre ne semble pas résider dans une interdiction, dans le LAE, de créer des suites secondaires dites «marquées », plus précisément des suites considérées moins optimales par exemple au niveau de la courbe de sonorité (par exemple [nv] dans neveu serait plus marqué que [vn] dans venait) ${ }^{3}$. En fait, toute suite consonantique secondaire autorisée dans le langage inter-adulte est également autorisée dans le LAE.

Puisque des variantes sans schwa, quel que soit le type de suite secondaire, sont présentes dans le LAE, on pourrait s'attendre à ce que l'enfant tente de produire ces variantes. Toutefois, puisque ce sont les variantes avec schwa qui sont le plus fortement présentes dans son input, on pourrait aussi s'attendre à ce que l'enfant se contente de produire ces variantes, considérées phonologiquement comme «non marquées » étant donné leur structure syllabique CVCV, et qu'il évite toute tentative de production des variantes sans schwa, également présentes dans le LAE mais avec une structure CCV et désormais considérées phonologiquement comme «plus marquées».

3. Au sujet de la marque, voir par exemple de Lacy (2006). 


\section{Le schwa dans le langage enfantin : méthodologie}

L'acquisition des variables linguistiques a suscité beaucoup d'intérêt ces dernières années et le consensus semble être à présent que la variation n'est plus «a by-product of the learning process, but an integral part of acquisition itself» (Roberts, 2005, p. 153-154). De plus, bien qu'il y ait un certain nombre de facteurs extralinguistiques qui différencient leur acquisition, les processus variables sont acquis en même temps que les processus catégoriels (voir l'étude de Smith, Durham \& Richards, 2013, sur l'acquisition de diverses variables grammaticales de l'anglais écossais). Sur la base de cette observation, nous estimons indispensable d'examiner l'acquisition du schwa à la lumière de celle d'autres phénomènes, tels que l'effacement syllabique et la création de suites consonantiques.

Nos questions de recherche nécessitent un grand nombre d'occurrences d'items schwa, aussi bien au niveau individuel qu'au niveau du corpus. Le schwa étant une variable phonologique, nous estimons primordial de relever non seulement les variantes préférées par l'enfant, avec ou sans schwa, mais aussi les variantes non préférées mais tout de même autorisées dans sa grammaire, avec ou sans schwa (voir la discussion dans Crain \& Wexler, 1999, sur la (non) grammaticalité de la variante non préférée). Deux hypothèses découlent de notre lecture des travaux antérieurs : la première est que l'enfant préfère la variante dont la complexité segmentale et syllabique est dite «moins marquée»; la deuxième est que l'enfant, dont le développement phonologique est plus avancé, a accès à une deuxième variante, qu'il n'emploie pas dans son langage spontané mais dont l'usage peut être encouragé lors d'une interaction avec un locuteur perçu comme moins disposé à s'adapter au niveau langagier de l'enfant: «[...] [i]t is, however, entirely possible that [selection] pressures do operate in situations unlike the situations we have sampled, for instance, away from home or with strangers.» (Brown, 1973, p. 412)

Relever le comportement du schwa au cours de l'acquisition nécessite la production multiple d'un ensemble contrôlé d'items schwa, qu'il aurait été relativement facile d'obtenir à l'aide d'images représentant ce type d'items. Il s'avère cependant que la situation formelle agit sur le comportement du schwa, du moins dans le langage adulte : les études dans Durand, Laks et Lyche (2009b) montrent bien par exemple que le schwa n'alterne que très peu dans le registre de la lecture. Cela nous a incitée à sélectionner d'autres stratégies d'enregistrement, à savoir l'enregistrement de données spontanées et de données semi-contrôlées. 
Premièrement, l'enregistrement de données spontanées permet non seulement la production d'un ensemble d'items schwa variés, mais il est également susceptible de dévoiler les variantes sélectionnées par défaut : si, pour un item schwa donné, l'enfant a accès à deux variantes, avec et sans schwa, on pourrait s'attendre à ce qu'il en préfère une dans son langage spontané (par exemple [vœny] et non pas [vny] pour venu). Ce type d'enregistrement est cependant problématique du fait qu'il ne permet pas d'assurer la production multiple d'un même item schwa, ni la production de données comparables. Deuxièmement, l'enregistrement de données semi-contrôlées facilite quant à lui la comparaison intra- et inter-locuteur, tout en étant susceptible de dévoiler un plus grand nombre de variantes phonologiques accessibles à l'enfant, y compris les variantes non préférées: si, pour un item schwa donné, l'enfant a accès à deux variantes tout en en sélectionnant une par défaut (par exemple [vœny]), il pourrait, dans une situation moins familière et face à de la variation dans l'input, être mené à produire la variante non préférée, également (par exemple [vny]). Ce deuxième type d'enregistrement est toutefois problématique de par le fait qu'il crée un contexte artificiel dans lequel on risquerait des productions ne reflétant qu'un simple mimétisme du stimulus adulte.

La solution semble résider dans la combinaison de ces deux stratégies d'enregistrement. Nous avons ainsi effectué un travail de terrain durant la première moitié de 2006, auprès de 13 enfants francophones résidant sur la côte lémanique en Suisse. Optant pour une observation longitudinale et transversale, nous avons suivi ces enfants - répartis en trois groupes d'âge - pendant 3-6 mois. Mentionnons ici que nous aurions voulu inclure un nombre égal d'enfants âgés de 2 ans, 2 ans et 6 mois et 3 ans au début de la période d'enregistrement, mais que, malheureusement, l'observation d'enfants de 2 ans a en partie échoué à cause d'un manque de locuteurs disponibles pendant la fenêtre temporelle réservée au travail de terrain.

Les données semi-contrôlées ont été obtenues à l'aide d'enregistrements des 13 enfants, effectués mensuellement à la crèche. Les données spontanées sont issues à l'aide d'enregistrements de 8 de ces 13 enfants, effectués hebdomadairement à domicile, le plus souvent avec la mère présente $^{4}$. Le tableau 1 fournit des renseignements supplémentaires sur le corpus.

4. Notons que Fabienne et Henri ont uniquement été enregistrés à la crèche. 


\begin{tabular}{|c|c|c|c|c|c|c|c|}
\hline $\begin{array}{c}\text { Groupe } \\
\text { d'âge }\end{array}$ & $\begin{array}{c}\text { Nom } \\
\text { enfant }\end{array}$ & Sexe & $\begin{array}{c}\text { Première } \\
\text { session }\end{array}$ & $\begin{array}{c}\text { Dernière } \\
\text { session }\end{array}$ & $\begin{array}{c}\text { Nom } \\
\text { mère }\end{array}$ & Spontané & $\begin{array}{c}\text { Semi- } \\
\text { contrôlé }\end{array}$ \\
\hline 1 & Fabienne & F & $2 ; 02.15$ & $2 ; 05.21$ & & 6 & 5 \\
\hline 1 & Henri & M & $2 ; 04.01$ & $2 ; 07.08$ & & 7 & 5 \\
\hline 2 & Lucas & M & $2 ; 07.01$ & $2 ; 10.25$ & Véronique & 16 & 5 \\
\hline 2 & Adèle & F & $2 ; 07.08$ & $2 ; 10.13$ & Valentine & 12 & 5 \\
\hline 2 & Janice & F & $2 ; 07.27$ & $3 ; 00.14$ & Nina & 15 & 6 \\
\hline 2 & Kim & M & $2 ; 08.29$ & $3 ; 00.05$ & & & 5 \\
\hline 2 & Théa & F & $2 ; 09.29$ & $3 ; 01.12$ & & & 5 \\
\hline 3 & Armand & M & $2 ; 11.13$ & $3 ; 04.03$ & Karoline & 17 & 6 \\
\hline 3 & Lambert & M & $2 ; 11.13$ & $3 ; 03.02$ & & & 5 \\
\hline 3 & Eric & M & $2 ; 11.16$ & $3 ; 02.15$ & & & 5 \\
\hline 3 & Albert & M & $3 ; 01.00$ & $3 ; 04.03$ & & & 5 \\
\hline 3 & Tom & M & $3 ; 01.17$ & $3 ; 06.05$ & Blanche & 19 & 6 \\
\hline 3 & Guy & M & $3 ; 02.14$ & $3 ; 07.06$ & Alice & 16 & 6 \\
\hline & & & & & \\
\hline
\end{tabular}

Tableau 1. - Présentation du corpus.

\section{Le schwa dans le langage enfantin : résultats}

Les enregistrements à domicile ont donné lieu à toute une série d'observations intéressantes. Premièrement, nous relevons une préférence globale pour la variante avec schwa, et ce dans les trois groupes d'âge (taux de présence général : $64 \%$, soit 1582/2 488 occurrences). Cette observation est intéressante du fait que la majorité des items schwa faisant partie du lexique de production des enfants sont sujets à un taux d'alternance très élevé dans le système cible, par exemple faisait $[\mathrm{f}(œ) \mathrm{z} \varepsilon] \rightarrow[\mathrm{f} œ z \varepsilon]$ (Tom 3;06.05). Un autre aspect intéressant est la production spontanée - rare, il est vrai - de variantes sans schwa cibles, avec absence vocalique et suite secondaire cible, attestées chez certains enfants des groupes d'âge 2 et 3 , par exemple remet [вœтв] (Tom 3;06.05) [втв] (Tот 3;04.19). Un troisième aspect intéressant est la production spontanée - encore plus rare - de variantes sans schwa non cibles, par exemple demi [d(œ)mi] $\rightarrow$ [mi] (Adèle 2;08.09), avec absence vocalique et réduction de la suite consonantique. Chez les enfants qui en produisent, la variante sans schwa non cible n'est pas accompagnée d'une deuxième variante avec schwa, ce qui nous mène à la deuxième observation principale tirée des données spontanées : dans la majorité des cas, les enfants emploient pour chaque item schwa une 
seule variante. Cela entraine une certaine variation lexicale à l'intérieur du corpus : bien que dans la majorité des cas, tous les enfants préfèrent la variante avec schwa, quel que soit l'item schwa en question, il arrive que, pour un mot donné, deux locuteurs du même groupe d'âge préfèrent chacun sa variante (2a). Il arrive aussi qu'un même locuteur préfère deux variantes différentes pour deux items schwa dont la structure phonotactique est la même (2b).

(2) Variation lexicale interne au corpus :

a. Variation inter-locuteur

$\begin{array}{lllll}\text { venu } & {[\mathrm{v}(œ) \mathrm{ny}]} & \rightarrow & \text { [vuny }] & \text { Armand }(3 ; 03.20) \\ \text { venu } & {[\mathrm{v}(œ) \mathrm{ny}]} & \rightarrow & {[\mathrm{vny}]} & \text { Guy }(3 ; 05.30)\end{array}$

b. Variation intra-locuteur

cheval $\quad\left[\int(\propto)\right.$ val $] \rightarrow$ [toval $]$ Adèle $(2 ; 09.23)$

cheveux $\left[\int(\propto) \mathrm{v} ø\right] \rightarrow[\mathrm{sjø}] \quad$ Adèle $(2 ; 08.22)$

Les enregistrements de données spontanées nous fournissent donc des renseignements sur les variantes que l'enfant sélectionne par défaut, sur l'éventuel taux d'alternance et finalement, sur la structure segmentale des variantes dans l'output.

Les enregistrements à la crèche ont donné lieu à une deuxième série d'observations intéressantes. Avant de passer aux résultats, nous présentons brièvement l'expérience développée pour cette étude. L'idée principale de l'expérience - outre la nécessité de collecter des données comparables - est d'inciter la production des variantes accessibles à l'enfant, y compris celles qui ne sont pas produites spontanément. Afin d'y parvenir, nous avons créé une présentation PowerPoint ${ }^{\circledR}$, dans laquelle sont intégrées des images illustrant, entre autres, des items schwa - y compris deux pseudo-mots chevir et fenasse ${ }^{5}$, qui partagent la structure phonotactique des items schwa cheval et fenêtre. Les images sont accompagnées de fichiers son pré-enregistrés, contenant des phrases lues par un locuteur romand natif. Chaque phrase qui contient un item schwa a été enregistrée avec deux variantes, avec et sans schwa, ce qui nous permet d'exposer l'enfant à la variante de l'item schwa qui n'est pas celle qu'il a produite auparavant.

5. Il a été porté à notre attention, par un des relecteurs, que fenasse existe en français. Cependant, le fait qu'il n'est pas listé dans Le Petit Robert, le Trésor de la langue française, ni sur Lexique.org, suggère qu'il s'agit d'un mot très peu courant et de ce fait inconnu des jeunes enfants. 
L'objectif de cette expérience est donc de tester si l'enfant, face à de la variation dans l'input immédiat, dévoile une deuxième variante à côté de la variante sélectionnée par défaut, et, si c'est le cas, d'identifier la structure segmentale de cette deuxième variante non préférée. La figure 2 illustre le déroulement de l'expérience.
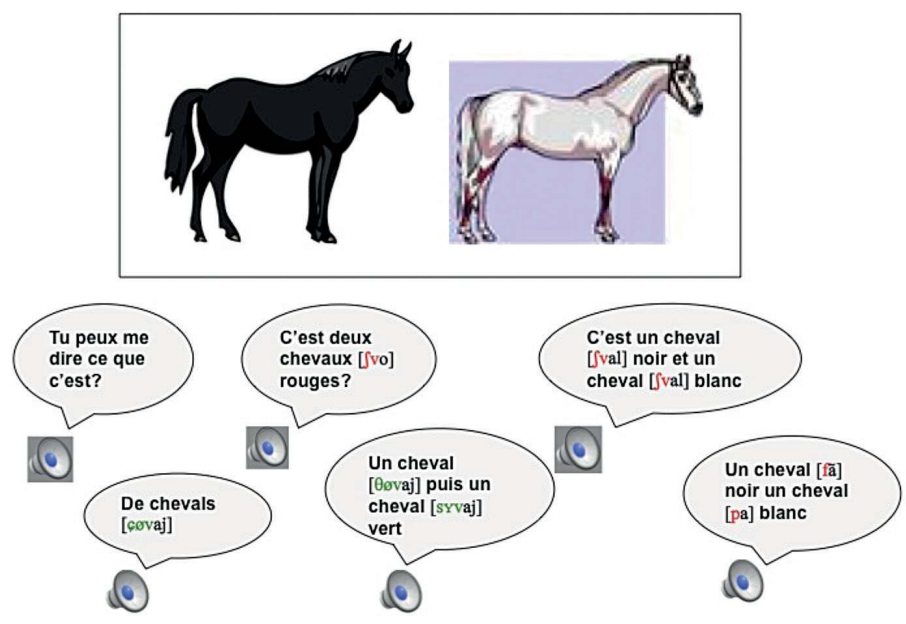

Figure 2. - Illustration de l'expérience PowerPoint . Interaction entre le locuteur romand natif (hautparleurs gris, ligne supérieure) et Kim (2;11.15).

Même si l'expérience a globalement bien fonctionné, il serait nécessaire d'apporter quelques modifications dans son déroulement en cas de réutilisation. Premièrement, la durée de l'expérience - nécessaire pour obtenir des données récurrentes à l'intérieur d'une même session - a suscité de l'impatience chez certains enfants et a même conduit parfois à une interruption prématurée de la session. Deuxièmement, bien que les données contiennent une grande quantité d'occurrences, le nombre limité d'items schwa choisis ne permet pas de nous prononcer sur les variantes non préférées ou non accessibles d'items schwa dont la structure phonotactique est autre que $\left[\int(œ) v\right]$ et $[f(œ) n]$. Troisièmement, les images ont parfois induit la production d'autres mots que ceux voulus, par exemple carré pour fenêtre, ou poulain pour petit cheval. Finalement, les pseudo-mots ont provoqué différentes réactions. Si certains enfants les acceptent d'emblée, d'autres les reproduisent au commencement mais reviennent à des mots connus plus tard dans la session, par exemple animal ou hibou au lieu de chevir. 
Malgré ces quelques défis, l'exposition à la variation a permis d'identifier les variantes accessibles à l'enfant. Premièrement, à l'instar des données spontanées, les données semi-contrôlées montrent une préférence globale pour la variante avec schwa (taux de présence général : $80 \%$, soit 1256/1571 occurrences). À nouveau, les données contiennent une certaine variation lexicale : par exemple, si Armand préfère la variante sans schwa de cheval dans son langage spontané (cf. note 1), le même patron se dessine lors de l'expérience. Notons ici un des avantages de l'aspect longitudinal de l'étude : vers la fin de la période d'enregistrement, à l'âge de 3;03.10, Armand tente la production dissyllabique du mot cheval, par exemple [çyval]. Deuxièmement, tous les enfants se révèlent sensibles à la variation dans l'input, à des degrés différents cependant : surtout attestée chez les enfants dont le développement phonologique est plus avancé, la sélection de la variante non préférée a lieu, dans la majorité des cas, après exposition à cette variante dans l'input, comme on le voit dans l'exemple (3) présenté ci-dessous.

(3) Scénario : image d'un cheval, puis d'un deuxième cheval :

$\begin{array}{lll}\text { Ça c'est un cheval } & \left.\text { [ } \int œ v a l\right] & \text { Albert }(3 ; 01.00) \\ \text { Oui, c'est un cheval. } & \text { [Jval] } & \text { locuteur romand natif } \\ \text { Et puis ça, c'est quoi? } & & \end{array}$

Un cheval qui court [joaj] Albert (3;01.00)

En outre, les enfants dont le développement phonologique est moins avancé, qui ne maitrisent pas ou mal les suites consonantiques, produisent occasionnellement des variantes sans schwa, toutefois non cibles, comme le montre l'exemple (4a). Également pour ce groupe d'enfants, la variante non préférée est observée, dans la plupart de cas, après exposition à cette variante dans l'input (voir l'exemple (4b)).

Tout en admettant que ces résultats doivent être confirmés par de futures recherches, nous suggérons que la réticence observée, auprès des jeunes, de sélectionner la variante dite "plus marquée» présentée dans l'input, est preuve que leur output passe par le filtre de leur grammaire, et qu'il ne s'agit pas de mimétisme. La nature manifestement développementale des modifications qu'ils emploient face aux rares cas d'absence vocalique renforce cette suggestion.

(4) Sélection de variantes sans schwa chez des enfants phonologiquement moins avancés :

a. Variantes sans schwa non cibles cheval $\left[\int(\infty)\right.$ val $] \rightarrow$ [təvat $] \sim[$ fwat $]$ Adèle $(2 ; 08.29)$ fenêtre $[\mathrm{f}(\mathrm{\infty}) \mathrm{n \varepsilon t \textrm {t }}] \rightarrow[\mathrm{frnn \varepsilon t}] \sim[\mathrm{n} \varepsilon] \quad$ Armand $(3 ; 01.21 / 3 ; 00.01)$ 
cerises [s(œ)віz] $\rightarrow$ [hajiç] [6і] Henri (2;05.06/2;05.27)

b. Scénario : commentaires sur deux images de fenêtres, puis une phrase spontanée

\begin{tabular}{|c|c|c|}
\hline $\begin{array}{l}\text { C'est tous les fenêtres } \\
\ldots \text { dans les fenêtres } \\
{[\ldots]}\end{array}$ & $\begin{array}{l}\text { [tınæt }] \\
{[\text { fnets }]}\end{array}$ & $\begin{array}{l}\text { Adèle }(2 ; 08.29) \\
\text { locuteur romand natif }\end{array}$ \\
\hline $\begin{array}{l}\text { ART fenêtre } \\
\text { Une fenêtre } \\
{[\ldots]}\end{array}$ & $\begin{array}{l}\text { [klæ:t] } \\
\text { [fnetь] }\end{array}$ & $\begin{array}{l}\text { Adèle }(2 ; 08.29) \\
\text { locuteur romand natif }\end{array}$ \\
\hline $\begin{array}{l}\text { Quand fais moi dodo } \\
\text { moi ma maman } \\
\text { PRo fermer la fenêtre }\end{array}$ & [กี๊กæ:t] & Adèle (2;08.29) \\
\hline
\end{tabular}

En plus de renforcer les patrons observés dans les données spontanées, les enregistrements de données semi-contrôlées nous fournissent donc des renseignements sur les variantes non préférées - et non accessibles chez les enfants qui n'alternent que très peu - ainsi que sur la structure segmentale de ces variantes dans l'output.

Avant de passer à la discussion, nous jugeons utile de nous attarder sur le traitement acoustique des occurrences des items schwa issus de notre corpus enfantin.

Traditionnellement, le schwa fait l'objet d'une classification dichotomique; soit il occupe une position nucléaire dans la structure syllabique, entouré de consonnes et sous la forme d'une voyelle articulatoirement proche de [œ] (ou [ø]), soit il est absent aussi bien du flux phonétique que de la structure syllabique, créant une suite consonantique secondaire.

Afin de saisir tout phénomène acoustique qui représente la syllabe schwa, opération qui à son tour nous permet de nous prononcer sur le traitement phonologique des items schwa employé par l'enfant, nous avons développé un système de codage qui va au-delà de la dichotomie présence-absence. Cette idée est en conformité avec les travaux récents qui révèlent une variabilité spectrale (Bürki, Racine, Andreassen, Fougeron \& Frauenfelder, 2008) aussi bien qu'une variabilité temporelle du schwa (Andreassen \& Racine, 2013, à paraitre; Bürki, Fougeron \& Gendrot, 2007; Bürki, Fougeron, Gendrot \& Frauenfelder, 2011), les travaux qui discutent des possibles traces que laisse le schwa absent dans le signal phonétique (Barnes \& Kavitskaya, 2002; Bürki, Fougeron, Veaux \& Frauenfelder, 2009; Côté \& Morrison, 2007; Fougeron \& Steriade, 1997), ainsi que le travail de Carter et Gerken (2004), qui montre une forte fidélité à la structure syllabique chez les enfants anglophones. 
Le tableau 2 affiche les huit structures d'output que nous avons relevées dans nos données. Trois structures, dont une deuxième interprétation phonologique qui est envisageable mais reste à déterminer, sont classées avec des points d'interrogation. Nous indiquons le taux d'usage des différentes structures par les pourcentages tirés des données semi-contrôlées.

\begin{tabular}{|c|c|c|c|}
\hline Structure d'output & $\begin{array}{l}\text { Présence } \\
\text { du schwa }\end{array}$ & $\begin{array}{c}\text { Absence } \\
\text { du schwa }\end{array}$ & $\begin{array}{c}\text { Taux } \\
\text { semi-contrôlé }\end{array}$ \\
\hline Voyelle pleine proéminente & $\sqrt{ }$ & & $\begin{array}{c}65 \% \\
(1024 / 1571)\end{array}$ \\
\hline Voyelle pleine non proéminente & $\sqrt{ }$ & & $\begin{array}{c}11 \% \\
(181 / 1571)\end{array}$ \\
\hline Voyelle réduite & $\sqrt{ }$ & $?$ & $\begin{array}{c}3 \% \\
(40 / 1571)\end{array}$ \\
\hline Redoublement de voyelle & $\sqrt{ }$ & & $\begin{array}{c}1 \% \\
(11 / 1571)\end{array}$ \\
\hline Suite secondaire contigüe & & $\sqrt{ }$ & \multirow{3}{*}{$\begin{array}{c}14 \% \\
(215 / 1571)\end{array}$} \\
\hline Suite secondaire décomposée & $?$ & $\sqrt{ }$ & \\
\hline Consonne allongée & $?$ & $\sqrt{ }$ & \\
\hline Suite secondaire réduite & & $\sqrt{ }$ & $\begin{array}{c}6 \% \\
(100 / 1571)\end{array}$ \\
\hline
\end{tabular}

Tableau 2. - Structures d'output pour les items schwa.

Nous donnons un exemple dans la figure 3 qui illustre la présence d'une voyelle dans la position du schwa. Deux aspects différencient la voyelle du schwa phonétique cible : il s'agit d'un redoublement de la voyelle finale, non suivi de consonne.

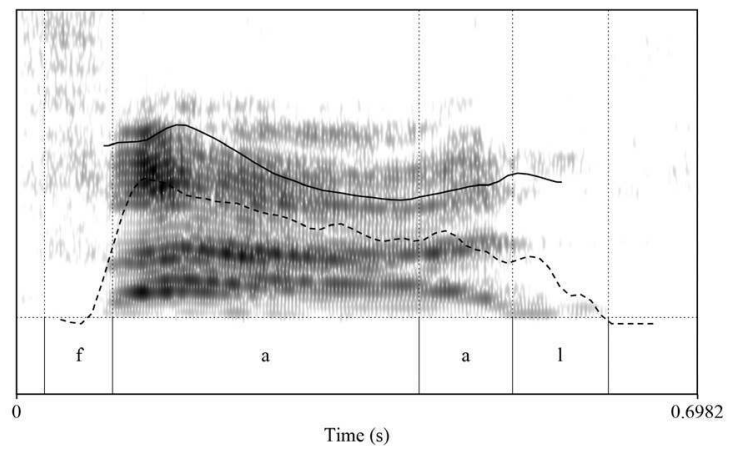

Figure 3. - Présence du schwa, redoublement de voyelle (cheval, Armand 2;11.21).

La ligne continue indique le pitch, la ligne pointillée, l'intensité. 
Afin de saisir toute stratégie articulatoire sélectionnée par l'enfant pour éviter la production d'une structure dite «marquée», nous avons opté pour une transcription très fine des syllabes schwa (présence ou absence), avec des symboles API non standards (ICPLA Executive Commitee, 1994). La figure 4 illustre la présence d'une voyelle réduite dans la position du schwa. La figure 5 illustre la présence d'une sonorante allongée dans cette position - la courbe mélodique montante étant l'indice de son statut syllabique. Notons que ces deux structures d'output sont attestées chez les enfants dont le développement phonologique est plus avancé, uniquement.

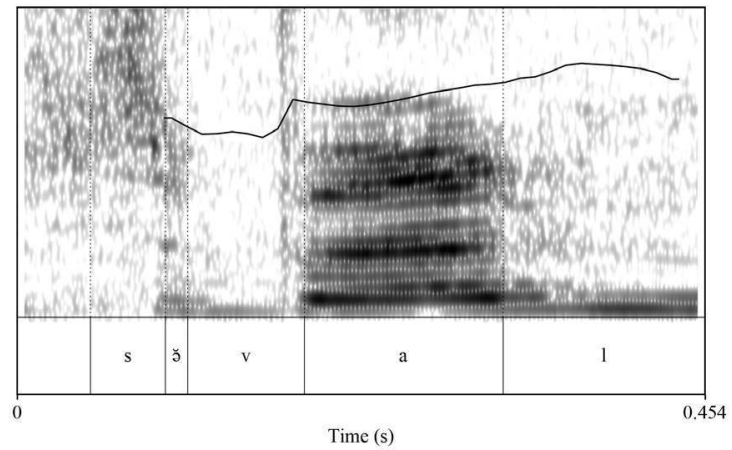

Figure 4. - Présence du schwa, voyelle réduite (cheval, Tom 3;05.16).

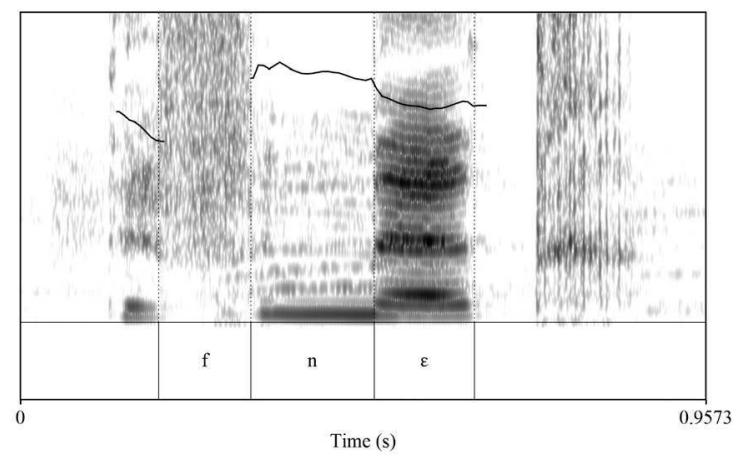

Figure 5. - Présence de la syllabe du schwa, sonorante allongée et syllabique (fenêtre, Tom 3;03.29).

Nous avons mentionné que, dans la situation semi-contrôlée, la réalisation d'une variante sans schwa non cible apparait surtout suite à l'absence de schwa dans l'input. Cette observation conduit à penser 
que les enfants disposent de moyens linguistiques pour produire une sorte d'alternance, même avant de maitriser pleinement l'effacement syllabique et les suites secondaires.

Dans l'exemple (5), présenté ci-dessous, nous proposons trois stades qui se dégagent de notre corpus de langage spontané et semi-contrôlé. Soulignons que l'alternance est présente à chaque stade, bien qu'à des degrés divers et sous différentes formes.

(5) Stades observés :

a. Voyelle pleine fortement préférée, effacement et substitution de consonne possible

Voyelle pleine cheval [厅(œ)val] $\rightarrow$ [syvaj] (Kim 2;11.15)

$>$ Modification (marginale) cheval $\left[\int(œ)\right.$ val $] \rightarrow[\mathrm{fa}](\mathrm{Kim} 2 ; 11.15)$

b. Voyelle pleine préférée, modification phonologique ou phonétique au niveau segmental possible

Voyelle pleine refais $[\mathrm{ь}(œ) \mathrm{f \varepsilon}] \rightarrow$ [ваfє] (Janice 2;10.25)

$>$ Voyelle réduite cheval $\left[\int(\infty)\right.$ val $] \rightarrow[$ ăwal] (Lambert 3;01.14)

$>$ Consonne syllabique regarde [в (œ)gard] $\rightarrow$ [ngaj] (Lucas 2;09.03)

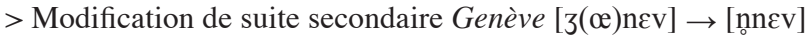
(Tom 3;06.01)

c. Voyelle pleine préférée, suite secondaire possible

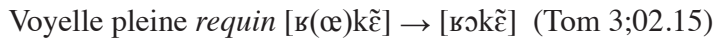

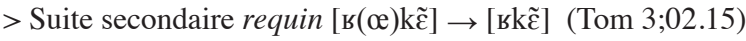

\section{Le schwa dans le langage enfantin : discussion}

Une des raisons pour lesquelles il existe très peu de travaux sur l'acquisition du schwa peut être la difficulté de collecter des données fiables : si les données spontanées n'assurent pas la production de toutes les variantes accessibles, le défi réside dans la façon de les recueillir. Divers travaux sur l'acquisition (p. ex. Brown, 1973; Crain \& Wexler, 1999) nous ont amenée à développer un scénario de dialogue, où le chercheur contrôle l'input de l'enfant. Le pré-enregistrement d'un locuteur romand natif assure l'exposition aux enfants des mêmes stimuli; un interlocuteur invisible, «statique» et non familier, toutefois engageant, qui produit des items schwa, avec ou sans schwa, selon la variante sélectionnée par l'enfant préalablement.

Puisque le LAE naturel contient davantage de formes non réduites que le langage inter-adulte, et que l'enfant, par conséquent, est possiblement moins enclin ou moins encouragé à produire des formes réduites 
dans sa parole spontanée, nous avons considéré qu'une conversation hors situation familiale pourrait plus facilement provoquer une gamme plus exhaustive de variantes accessibles aux enfants : toujours de manière indirecte, et sous forme ludique, nous avons donc invité l'enfant à dévoiler ses capacités linguistiques - un choix méthodologique qui a apporté des données permettant de nous prononcer enfin sur les contraintes qui semblent agir sur l'output phonologique de l'enfant.

La combinaison de deux stratégies d'enregistrement, spontanée et semi-contrôlée, s'est donc montrée fructueuse dans cette première étude détaillée visant l'acquisition de l'alternance du schwa. En premier lieu, nous observons que le schwa alterne peu chez les enfants âgés de 2,5 à 3,5 ans. Cela indique que les enfants sont avant tout guidés par des contraintes intra-grammaticales plutôt que par des contraintes extragrammaticales, telles que la fréquence des variantes dans la langue ambiante. À ce propos, il faut cependant rappeler que la tendance à plus forte présence du schwa dans le LAE pourrait renforcer la préférence observée chez les enfants.

En second lieu, nous observons, dans la situation semi-contrôlée, que, dans certains cas, une deuxième variante est accessible, quoique non préférée dans le langage spontané. Ces deux observations appellent quelques réflexions quant à la réutilisation de la méthodologie choisie.

Premièrement, le corpus de données spontanées montre que les enfants produisent un nombre d'items schwa variés très limité. En comparant avec nos données inter-adultes tirées du corpus PFC, nous observons que, si les adultes produisent 111 items schwa par heure d'enregistrement (14 heures de parole spontanée au total), en moyenne, les enfants n'en produisent que 54 (46 heures de parole spontanée au total). Si ce petit nombre est représentatif pour l'usage quotidien de l'enfant, il semble évident que le taux de production d'items schwa est plus faible dans le langage enfantin comparé à celui du langage adulte. Avant d'aboutir à cette conclusion, il faudrait néanmoins chercher d'autres façons d'observer l'usage des items schwa, ainsi que la variation phonologique émergente, par exemple l'enregistrement intensif d'un nombre plus restreint d'enfants, à domicile dans différentes situations, sans la présence du chercheur (voir Smith, Durham \& Fortune, 2007, 2009; Tomasello \& Stahl, 2004).

Deuxièmement, les enfants témoignent d'une richesse stratégique dans leur traitement des items schwa, richesse qui, à son tour, nous guide dans la recherche des facteurs entravant l'alternance de schwa telle qu'elle se manifeste dans le système cible. Deux contraintes émergent 
des données : être fidèle au nombre syllabique et ne pas créer de suites secondaires. La première contrainte est illustrée par la présence quasiobligatoire du schwa, non seulement dans des structures syllabiques $\mathrm{CVCV}$, telles que par exemple besoin $[\mathrm{b}(œ) \mathrm{zw} \tilde{\varepsilon}] \rightarrow[$ bœe $\tilde{\varepsilon}]$ (Armand $3 ; 04.00)$, mais aussi dans des structures où la présence du schwa est accompagnée d'un effacement consonantique, par exemple Genève [3(œ)nعv] $\rightarrow$ [incf] (Fabienne 2;05.21). Si ce dernier exemple indique que la présence du schwa n'est pas nécessairement un moyen d'éviter des suites consonantiques, la production occasionnelle d'une voyelle phonétique non cible, quant à elle, confirme que la réalisation de la syllabe prime sur la fidélité qualitative, par exemple petit cheval $[\mathrm{p}(\propto)$ ti $\left.\int(œ) v a l\right] \rightarrow$ [piti fəvaj] (Henri 2;04.01). La deuxième contrainte est illustrée par l'observation que les suites secondaires dites «plus marquées» sont évitées. Les enfants dont le développement phonologique est plus avancé maitrisent les suites primaires et produisent les suites secondaires de même type sans difficultés articulatoires considérables, par exemple [vn] à sonorité croissante dans venir, $\left[\int \mathrm{v}\right]$ avec sibilante initiale dans cheveux. Les mêmes enfants tentent cependant moins la production de suites secondaires à sonorité décroissante, par exemple [вғ] dans refaire, [ви] dans renard.

La richesse stratégique dont les enfants se servent pour éviter des outputs moins optimaux ressort avant tout de la situation spontanée - hors de portée du contrôle du chercheur. Notre proposition que deux contraintes sont actives dans le traitement d'items schwa bénéficierait donc d'une expérience contrôlée qui teste l'impact de l'une et l'autre au cours de l'acquisition.

Troisièmement, notre corpus de données enfantines contient de la variation inter-locuteur - à travers et à l'intérieur des groupes d'âge. Afin de saisir le parcours acquisitionnel, et afin de confirmer et expliquer l'usage des stratégies variées employées sur les items schwa aux différents stades, la poursuite de cette recherche nécessiterait l'enregistrement d'un corpus élargi et équilibré, couvrant les tranches d'âge déjà recueillies, aussi bien que celles qui leur sont inférieures et supérieures. Un tel corpus permettrait non seulement l'analyse statistique des données, mais également l'analyse qualitative d'une gamme de grammaires individuelles - avec leurs similitudes et idiosyncrasies.

Pour conclure ce travail, nous soulignons premièrement que des données de corpus apportent un début de réponse à la question du comportement du schwa lors du parcours acquisitionnel. Deuxièmement, la combinaison de méthodes permet d'identifier les variantes phonolo- 
giques accessibles et celles qui sont préférées, ce qui à son tour permet d'entreprendre une analyse phonologique des stratégies qu'emploie l'enfant afin d'éviter des structures dites «marquées». Finalement, la récolte de données dans différents contextes de communication permettra, dans de futures recherches, d'entamer la discussion sur les stratégies employées par l'enfant en face de différents types d'interlocuteurs.

\section{Remerciements}

Ce travail a bénéficié des commentaires et remarques judicieuses de Chantal Lyche (Université d'Oslo) et d'Isabelle Racine (Université de Genève) et de quatre relecteurs que je tiens à remercier ici. Cette étude n'aurait pas été possible sans la bienveillance et la disponibilité des locuteurs, à qui je souhaite exprimer ici toute ma gratitude.

\section{RÉFÉRENCES BIBLIOGRAPHIQUES}

Andreassen, Helene N. (2013). Schwa: Distribution and Acquisition in Light of Swiss French Data (Thèse de doctorat). Université de Troms $\varnothing$, Troms $\varnothing$. Disponible en ligne sur <http://hdl.handle.net/10037/5193>

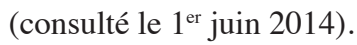

Andreassen, Helene N. \& Racine, Isabelle. (2013, décembre). Schwa et variation inter-régionale : une analyse de trois points d'enquête suisses. Communication présentée aux Journées PFC «Regards croisés sur les corpus oraux », Paris, France.

AndREASSEn, Helene N. \& Racine, Isabelle. (À paraitre). Variation in Switzerland: The Behaviour of Schwa in Martigny, Neuchâtel and Nyon. Dans S. Detey, J. Durand, B. Laks \& C. Lyche (dir.), Varieties of Spoken French: A Source Book. Oxford : Oxford University Press.

Barnes, Jonathan \& Kavitskaya, Darya. (2002). Phonetic Analogy and Schwa Deletion in French. Berkeley Linguistic Society, 28, 39-50.

BAZYLKo, Sławomir. (1976). Groupes consonantiques primaires et secondaires à l'initiale du mot dans le français contemporain. La Linguistique, 12(1). 63-80.

Brown, Roger. (1973). A First Language: The Early Stages. Cambridge, Mass. : Harvard University Press.

Bürki, Audrey, Fougeron, Cécile \& Gendrot, Cédric. (2007). On the Categorical Nature of the Process Involved in Schwa Elision in French. Dans Proceedings of Interspeech 2007 (p. 1026-1029). Disponible en ligne sur <www.isca-speech.org/archive/interspeech_2007/i07_1026. html $>$ (consulté le $1^{\text {er }}$ juin 2014). 
Bürki, Audrey, Fougeron, Cécile, Gendrot, Cédric \& Frauenfelder, Ulrich H. (2011). Phonetic Reduction versus Phonological Deletion of French Schwa: Some Methodological Issues. Journal of Phonetics, 39(3), 279-288. <http://dx.doi.org/10.1016/j.wocn.2010.07.003>.

Bürki, Audrey, Fougeron, Cécile, Veaux, Christophe \& Frauenfelder, Ulrich H. (2009). How Similar Are Clusters Resulting from Schwa Deletion in French to Identical Underlying Clusters? Dans Proceedings of Interspeech 2009 (p. 2271-2274). Disponible en ligne sur <www. isca-speech.org/archive/interspeech_2009/i09_2271.html> (consulté le $1^{\text {er }}$ juin 2014).

Bürki, Audrey, Racine, Isabelle, Andreassen, Helene N., Fougeron, Cécile \& FRAUENFELDER, Ulrich. (2008). Timbre du schwa en français et variation régionale : une étude comparative. Dans Actes des XXVII ${ }^{\text {es }}$ Journées d'études sur la langue (p. 1-4). Avignon. Disponible en ligne sur

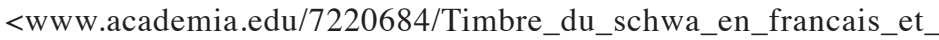
variation_regionale_Une_etude_comparative> (consulté le $1^{\text {er }}$ juin 2014).

Carter, Allyson \& Gerken, LouAnn. (2004). Do Children's Omissions Leave Traces? Journal of Child Language, 31(3), 561-586. <http://dx. doi.org/10.1017/S030500090400621X>.

Côté, Marie-Hélène \& Morrison, Geoffrey Stewart. (2007). The Nature of the Schwa/Zero Alternation in French Clitics: Experimental and NonExperimental Evidence. French Language Studies, 17(2), 159-186. <http://dx.doi.org/10.1017/S0959269507002827>.

Crain, Stephen \& Wexler, Kenneth. (1999). Methodology in the Study of Language Acquisition: A Modular Approach. Dans W. C. Ritchie \& T. K. Bhatia (dir.), Handbook of Child Language Acquisition (p. 387425). San Diego : Academic Press.

De Lacy, Paul. (2006). Markedness. Reduction and Preservation in Phonology. Cambridge : Cambridge University Press.

Durand, Jacques, LAKs, Bernard \& Lyche, Chantal. (2002). La phonologie du français contemporain : usages, variétés et structure. Dans C. D. Pusch \& W. Raible (dir.), Romanistische Korpuslinguistik - Korpora und gesprochene Sprache / Romance Corpus Linguistics - Corpora and Spoken Language (p. 93-106). Tübingen : Gunter Narr Verlag.

Durand, Jacques, LAKs, Bernard \& Lyche, Chantal. (2009a). Le projet PFC (phonologie du français contemporain) : une source de données primaires structurées. Dans J. Durand, B. Laks \& C. Lyche (dir.), Phonologie, variation et accents du français (p. 19-62). Paris : Hermès.

Durand, Jacques, Laks, Bernard \& Lyche, Chantal (dir.). (2009b). Phonologie, variation et accents du français. Paris : Hermès.

Fikkert, Paula, Levelt, Clara C. \& van De Weijer, Joost. (2005). Input, Intake and Phonological Development: The Case of Consonant Har- 
mony. Manuscrit. Radboud University Nijmegen, Leiden University and the University of Lund. Disponible en ligne sur <www.fikkert.com/ Publications/8.\%20FLSubmit.pdf> (consulté le $1^{\text {er }}$ juin 2014).

Fougeron, Cécile \& Steriade, Donca. (1997). Does Deletion of French Schwa Lead to Neutralization of Lexical Distinctions? Dans G. Kokkinakis, N. Fakotakis \& E. Dermatas (dir.), Proceedings of Eurospeech 97 (vol. 2, p. 943-946). Disponible en ligne sur <www.researchgate. net/profile/Fougeron_Cecile/publications/2> (consulté le $1^{\text {er }}$ juin 2014).

Foulkes, Paul, Docherty, Gerard \& Watt, Dominic. (2005). Phonological Variation in Child-Directed Speech. Language, 81(1), 177-206. Disponible en ligne sur <www.jstor.org/stable/4489857> (consulté le $1^{\text {er }}$ juin 2014).

ICPLA Executive Commitee. (1994). The extIPA Chart. Journal of the International Phonetic Association, 24(2), 95-98. <http://dx .doi.org/10. 1017/S0025100300005107>.

LIEGEOIS, Loïc. (2014). Usage des variables phonologiques dans un corpus d'interactions naturelles parents-enfant: impact du bain linguistique et dispositifs cognitifs d'apprentissage (Thèse de doctorat). Université Blaise-Pascal - Clermont-Ferrand.

LiÉgeois, Loïc, SAdDour, Inès \& Chabanal, Damien. (2012). L'élision du schwa dans les interactions parents-enfant : étude de corpus. Dans Actes de la conférence conjointe JEP-TALN-RECITAL 2012 (vol. 1, p. 313320). Disponible en ligne sur <http://aclweb.org/anthology/F12-1040> (consulté le $1^{\text {er }}$ juin 2014).

MARTINET, André. (1971). La prononciation du français contemporain : témoignages recueillis en 1941 dans un camp d'officiers prisonniers. Genève : Droz.

RACINE, Isabelle. (2008). Les effets de l'effacement du schwa sur la production et la perception de la parole en français (Thèse de doctorat). Université de Genève, Genève. Disponible en ligne sur <http://archiveouverte.unige.ch/unige:602> (consulté le $1^{\text {er }}$ juin 2014).

Roberts, Julie. (1997). Acquisition of Variable Rules: A Study of (-t, d) Deletion in Preschool Children. Journal of Child Language, 24(2), 351372.

Roberts, Julie. (2005). Acquisition of Sociolinguistic Variation. Dans M. J.Ball (dir.), Clinical Sociolinguistics (p. 153-164). Malden, MA : Blackwell.

Smith, Jennifer, Durham, Mercedes \& Fortune, Liane. (2007). "Mam, My Trousers Is Fa'in Doon!": Community, Caregiver, and Child in the Acquisition of Variation in a Scottish Dialect. Language Variation and Change, 19(1), 63-99. <http://dx.doi.org/10.1017/S0954394 507070044>. 
Smith, Jennifer, Durham, Mercedes \& Fortune, Liane. (2009). Universal and Dialect-Specific Pathways of Acquisition: Caregivers, Children, and t/d Deletion. Language Variation and Change, 21(1), 69-95. <http:// dx.doi.org/10.1017/S0954394509000039>.

Smith, Jennifer, Durham, Mercedes \& Richards, Hazel. (2013). The Social and Linguistic in the Acquisition of Sociolinguistic Norms: Caregivers, Children, and Variation. Linguistics, 51(2), 285-324. <10.1515/ling2013-0012>.

Tomasello, Michael \& Stahl, Daniel. (2004). Sampling Children's Spontaneous Speech: How Much Is Enough? Journal of Child Language, 31(1), 101-121. <http://dx.doi.org/10.1017/S0305000903005944>.

Vosoughi, Soroush. (2010). Interactions of Caregiver Speech and Early Word Learning in the Speechome Corpus: Computational Explorations (Mémoire de master, MIT). Disponible en ligne sur <http://hdl.handle. net/1721.1/62082> (consulté le $1^{\text {er }}$ juin 2014).

WALTER, Henriette. (1982). Enquête phonologique et variétés régionales du français. Paris : Presses universitaires de France. 